\title{
Genetic Influences on Four Measures of Executive Functions and Their Covariation with General Cognitive Ability: The Older Australian Twins Study
}

Running head: Genetic influences on executive function and general cognitive ability in older adults

Teresa Lee ${ }^{1,2}$, Miriam A. Mosing ${ }^{3,4}$, Julie D. Henry ${ }^{4}$, Julian N. Trollor ${ }^{1,5}$, David Ames ${ }^{6,7}$, Nicholas G. Martin $^{3,4}$, Margaret J. Wright ${ }^{3,4}$, Perminder S. Sachdev ${ }^{1,2}$, OATS Research Team ${ }^{1}$

${ }^{1}$ Brain and Aging Research Program, School of Psychiatry, Faculty of Medicine, University of New South Wales, Australia

${ }^{2}$ Neuropsychiatric Institute, Prince of Wales Hospital, Sydney, Australia.

${ }^{3}$ Genetic Epidemiology Unit, Queensland Institute of Medical Research, Brisbane, Australia

${ }^{4}$ School of Psychology, University of Queensland, Brisbane, Australia

${ }^{5}$ Department of Developmental Disability Neuropsychiatry, School of Psychiatry, Faculty of Medicine, University of New South Wales, Australia

${ }^{6}$ National Aging Research Institute, University of Melbourne, Australia

${ }^{7}$ Department of Aging and Health, University of Melbourne, Australia

Corresponding author:

Teresa Lee

Neuropsychiatric Institute, Euroa Centre, Prince of Wales Hospital, Randwick, New South Wales, Australia

Tel: 612 93823739, Fax: 612 93823774, Email: teresa.lee@unsw.edu.au 


\begin{abstract}
"Executive Functions" is a multidimensional construct which encompasses many higherorder cognitive control operations, and is considered a potential mediator of age-associated changes in other cognitive domains. Here we examine the heritability of four measures of Executive Functions (EF), and the genetic influences on their covariation with General Cognitive Abilities (GCA) from the Older Australian Twins Study (OATS). Participants included 117 pairs of monozygotic twins, 98 pairs of dizygotic twins, and 42 single twins, with a mean age of 71 . Genetic modeling showed that additive genetic factors contributed to $59 \%, 63 \%, 29 \%$, and $31 \%$ of the variance in the four measures: working memory, verbal fluency, response inhibition and cognitive flexibility, respectively. The phenotypic associations among the four EF measures were modest, which is in line with other evidence that $\mathrm{EF}$ is a multi-dimensional construct. All of the covariation between the EF measures was attributable to a common genetic factor. Similarly, all of the covariation between EF and General Cognitive Ability was explained by a common genetic factor, with no significant covariance due to environmental (E) factors. The genetic correlations between the measures were moderately high, suggesting that they may have common biological underpinnings. The genetic influence in the covariation of the EF measures and GCA also suggests that some aspects of EF and GCA share the same genes or same set of genes.
\end{abstract}

Keywords: Genetic influence, Twins, Aging, Executive Function, General Cognitive Ability 
Executive Functions (EF) comprise abilities that enable an individual to respond in an adaptive way to novel situations, and they serve as the basis of many cognitive, emotional, and social skills (Lezak et al. 2004). It is a multidimensional construct which encompasses higher order control operations, such as planning, organising, problem-solving, initiation of action, monitoring, sequencing, flexibility in thinking, formulating goals, working memory, generating response alternatives, decision-making and judgment (Smith and Jonides 1999; West 1996). It has been conceptualised as a "superordinate" or "meta" cognitive activity by many (Baddeley 1986, 1990; Shallice 1998), as it serves to control and integrate other cognitive activities (Baddeley 1990).

A common feature of these cognitive abilities is that they are closely linked to the integrity of prefrontal and striatal brain structures (Kimberg and Farrah 1993). Specifically, neuroimaging studies indicate that the brain changes associated with aging occur earlier and progress particularly rapidly in the prefrontal areas implicated in EF (Fjell et al. 2009, Head et al. 2008; Raz et al. 1998). These data provide support for the frontal-executive theory of aging (West, 1996), in which it argued that age-related changes in cognitive functions can be explained by early and localised changes in the frontal lobes, and that reductions in executive control processes contribute to age-related changes in other cognitive abilities such as memory and reasoning (see also; Moscovitch and Winocur 1992). This theoretical perspective therefore differs from the other preeminent model of cognitive aging, which attributes age-related changes in cognitive function to changes in general resource parameters such as processing speed (Salthouse, 1996). 
Given the central role of EF in cognition, it is important to examine the sources of variation among individuals. Research suggests that heritability estimates may vary as a function of the specific measure used to index EF, as well as the age of the sample. For example, the heritability for Stroop interference was $50 \%$ for 12 years old twins (Stins et al. 2004), 34\% for a sample with age ranging from 40 to 70 (Johnson et al. 2003), and 14\% for mean age of 23 (Taylor 2007). In contrast, performance on the Wisconsin Card Sorting Test was not influenced by genetic factors in the latter study (Taylor 2007). In a study involving 17 year-old twins, Friedman et al. (2008) reported that the three executive control processes of "inhibition", "updating" (working memory) and "set shifting" (mental flexibility) were one of the most heritable cognitive traits, with a highly heritable common factor (99\%) and there was also additional specific genetic influence to each of the particular EF processes.

Few studies have examined executive functioning in older adult twins. Swan and Carmelli (2002) examined verbal fluency, Digit Symbol, Stroop Inhibition and Trail Making Test B in male twins (mean age 71years) who were members of the National Heart, Lung, and Blood Institute (NHLBI) Twin Study, and reported a high heritability of 79\% for the "shared executive control factor" derived from these measures. Another study by the same group (Lessov-Schlaggar et al., 2007) reported heritability estimates of $83 \%, 61 \%$, and $43 \%$ for Digit Symbol, Stroop Inhibition, and Trail Making Test B, respectively. Similarly, Giubilei et al. (2008) reported high heritability estimates for EF measures in a sample including both sexes (mean age 67), $79 \%$ for selective attention and $62 \%$ for verbal generation. 
The investigation of the heritability of EF should be understood in the context of genetic influences in general cognitive functioning. However, to our knowledge, the genetic influences in the covariation of general cognitive ability and other cognitive domains such as EF in older adults have not been examined, except for our recent study (Lee et al. 2011) on the genetic influence on the covariation of processing speed and general cognitive ability (GCA). Investigations into genetic influences on variation in GCA in older adults have been reported by the Swedish Adoption and Twin Study of Aging (SATSA). Heritability of GCA was $81 \%$ at mean age of 65 (Pedersen et al. 1992; Plomin et al. 1994), and 62\% at the mean age of 80 (McClearn et al. 1997), while our study recent study reported a heritability estimate of GCA to be $74 \%$.

Given the relatively few studies on the genetic influences on measures of EF in the elderly, we aimed to extend this literature by exploring genetic and environmental influences on four EF measures and their covariation with general cognitive ability (GCA) in the Older Australian Twins Study. We included four commonly used measures of EF that tap Working Memory, Response Generation, Response Inhibition, and Cognitive Flexibility respectively; our General Cognitive Ability factor comprised verbal abstract reasoning ability, visuospatial problem-solving skills, and an episodic memory composite. The aims of this study were: first, to investigate the heritability of each of the four EF measures; and second, to examine the genetic influence on the covariation between the EF measures and General Cognitive Ability.

\section{Methods}


The methodology of the OATS has been described in detail in Sachdev et al. (2009). The sample of participants included was the same as our recent study on the genetic influence on measures of processing speed and the covariation with GCA (Lee et al. 2011), and is briefly summarised here.

Participants

Monozygotic (MZ) and dizygotic (DZ) twin pairs, aged 65 and above, were recruited from the Australian Twin Registry (ATR). According to a recent estimate, there are 1,226 pairs of MZ twins and 1,423 pairs of DZ twins aged 65 and older registered with the ATR. More than $90 \%$ of these twin pairs reside in the three Eastern states of Australia (New South Wales, Victoria, and Queensland), where the OATS sample was drawn. Participants were also recruited through advertisements, media, clubs, and older citizens' networks (Sachdev et al. 2009).

The study sample consisted of the first 472 OATS participants, comprising 117 pairs of MZ twins, 98 pairs of DZ twins, and 42 individuals (whose co-twin had not been assessed at the time of data collection), with a mean age of 71 and a standard deviation (sd) of 5.2 (range $65-88)$, and an average of $11(\mathrm{sd}=3)$ years of education. There was an over-representation of females, with a ratio of 2:1, and 17 participants were from a Non-English speaking background. The inclusion criteria were: ability to consent to participate, have a consenting co-twin, having completed some education in English, being of at least low average intelligence (NART-IQ $\geq 80$ ), and with a Mini-Mental State Examination (Folstein and Folstein 1975) score of $\geq 24$. Exclusion criteria were life-threatening illness, acute psychosis, and inadequate English to participate in assessment. Zygosity was determined by the participants' responses to a questionnaire about physical similarities of a twin pair. For a sub- 
sample (88 MZ pairs, 79 DZ pairs) who was recently genotyped with high-density SNP arrays, correct zygosity assignment was ascertained to be $100 \%$.

Measures

The following four measures of EF were included:

(1) Working memory was measured using Digit Span Backward (Wechsler 1997). The trial with the longest series of numbers correctly repeated backwards was the score. This task imposes demands on working memory, and is distinct from Digit Span Forward, a more passive span of attention (Lezak et al. 2004). Digits Span Backwards alone (without Digit Span Forward) was used or considered as a measure of working memory or executive function in the following studies: Bugaiska et al. (2006), and Bunce and McCready (2005); Cirulli et al. (2010), Luciano et al. (2009). This measure will be referred to as WorkMem hereafter.

(2) Response generation (Verbal Fluency) was measured using the Controlled Oral Word Association Test (COWAT; Benton 1967). The total number of words generated in one minute starting with each of the three designated letters F, A, and S was the score. This measure of verbal fluency has been widely used to index executive dysfunction (Byran and Luszcz 2000), and will be referred to as "Fluency" from here onwards.

(3) Response inhibition was measured by Stroop Colour and Word Test Parts 1 and 3 (DelisKaplan, 2001). This test was modified to form part of the in-house computerised battery (Sachdev et al. 2010). Part 1 required the naming of colours and Part 3 required naming of colour names printed in a discordant ink colour. The ratio of the time taken to complete 
Stroop 3 and Stroop 1 (3/1) indexed response inhibition (Lansbergen et al. 2007), as in Henry et al. (2009), and will be referred to as "Inhibition".

(4) Cognitive flexibility was measured by the Trail Making Test A and B (Reitan and Wolfson 1985). Part A required drawing lines to connect a series of consecutive numbers, while Part B required drawing lines, switching between numbers and letters. The ratio of the time taken to complete the two parts (B/A) (as in Arbuthnott and Frank, 2000) was the score. In this way, we have partialled out the time (speed) component of the task, and will be referred to as "Flexibility".

The availability of data for each EF variable differed, being over $99 \%$ for Fluency and WorkMem, $97.5 \%$ for Flexibility, $70 \%$ for Inhibition. The majority of missing data (for Inhibition) was due to technical failure with computerised testing and a minority due to participants' visual difficulties.

A general cognitive ability (GCA) measure was derived from the following:

(1) Similarities (WAIS-III, Wechsler 1997) a test of verbal abstract reasoning ability, which required abstracting the similarity of common objects or concepts.

(2) Block Design (WAIS-R, Wechsler 1981) required constructing models using blocks to match the geometric patterns printed on a stimulus booklet, a test of visuo-spatial and problem-solving skills. 
(3) A composite measure of episodic memory obtained from three sources: immediate and delayed recall of Story A of the Logical Memory subtests (Wechsler Memory Scale-Revised Wechsler 1987); the number of words recall at the last of five trials, delayed recall, total learning scores from the Rey Auditory Verbal Learning Test (Rey 1964); and the Benton Visual Retention Test (Sivan and Spreen 1996), a test of visual recognition memory in a multiple-choice format.

In including two measures of intellectual functions and three measure of episodic memory to form a GCA composite, we are in line with the SATSA reports (Pedersen et al.'s 1992) that have included verbal, visual, speed, and memory measures in their First Principal Component of General Cognitive Ability. The GCA score was then transformed to an IQ equivalent, with a mean of 100 and a standard deviation of 15 . Data from four participants were identified as outliers: one from each of Similarities, Block Design, the verbal list learning test and the visual memory test. The GCA measure was available for $93.4 \%$ of the sample.

In addition, in order to ascertain that any genetic and environmental covariation between GCA and EF was not due to overlap in measurement of some aspects of GCA and EF involving frontal and executive functions, we used a measure to estimate general intellectual functioning, the National Adult Reading Test (NART; Nelson and Willison 1991). The NART required oral reading of 50 phonetically irregular words, and an equivalent IQ score could be derived from the error score. This test provided an estimate of intellectual functioning that is relatively independent of frontal and executive functions. 
Statistical analyses

Values that were more than three standard deviations away from the variable mean were Winsorised. All EF variables were square-root transformed to correct for skewness, except for Fluency, which was normally distributed. Scores for Flexibility and Inhibition were reversed to be consistent with the scores from Fluency and WorkMem so that the higher the score the better participants' performance. In order to facilitate comparison of variance, all variable scores were transformed to z-scores (mean of zero and a variance of one). Prior to genetic modeling, each of the variables was tested for equality of means, within twin pairs and across the two zygosity groups, as well as for equality of variance. No significant difference was found in the variable means within twin pairs or across zygosity groups (all $p \mathrm{~s}$ >.05). Age was included as a fixed covariate for genetic modeling as it is a recognised risk factor in age-related diseases. Sex was also included as a fixed covariate because males and females have demonstrated advantage/disadvantage on different cognitive tests, such as Digit Symbol (Snow and Weinstock 1990).

The classical twin design was used to estimate the genetic and environmental influences on the variation in and covariation between measures. While MZ twins share all their genetic endowment, DZ twins only share on average $50 \%$ of their segregating DNA. The twin design uses this information on the genetic relatedness of twins and allows for the proportioning of variance into additive genetic (A) and environmental influences. Environmental factors are either "shared" between the twin pair (C) and include environment or events that are experienced similarly by the twins and make them more similar to each other, or "non- 
shared" (E, which includes measurement error) which reflect experiences unique to one of the twins, such as an acquired brain injury in one twin. While " $\mathrm{C}$ " influences increase a twin pair's resemblance in one trait, E influences make the twins more distinct from one another. "A" influences are suggested if the MZ twin correlation is larger than the DZ twin correlation, and "C" is indicated when the $\mathrm{DZ}$ correlation is more than half the MZ correlation. An important assumption of the classical twin design is that MZ and DZ twins only differ in terms of genetic relatedness, that is, "C" influences are not different for $\mathrm{MZ}$ compared to DZ twins (Purcell, 2008).

Multivariate genetic modeling was conducted to examine genetic and environmental influences on the relationship between the four EF measures and GCA, using the full information maximum likelihood (FIML) estimation in Mx (Neale et al. 2002; Neale, 2005). This procedure makes use of both paired and single twins, the latter contributing to estimates of means and variances. The two groups (MZ and DZ twins) each have two sets of means, one for each twin, for each of the phenotypes. A Cholesky decomposition was fitted first, estimating all parameters, with variation in each measure attributed to either common or specific additive genetic (A) and environmental ( $\mathrm{C}$ and $\mathrm{E})$ factors. This was followed by progressively more restricted models that were compared to the fit of the previous model. In Maximum Likelihood procedures, the -2LL (minus two times log-likelihood) statistics are compared between the saturated model and the reduced models to determine the most parsimonious model. The order of variables entered was: GCA, WorkMem (largest phenotypic correlation with GCA), Fluency (most heritable EF measure), Inhibition, and Flexibility. 


\section{Results}

The means and standard deviations for the four EF variables and GCA are shown in Table 1. No significant difference was found in the means within twin pairs or across zygosity groups (all $p \mathrm{~s}>.05$ ). A decrease in GCA was found with increasing age, and females performed better on Fluency and males performed better on WorkMem.

\section{Table 1 about here}

\section{Phenotypic Correlations}

The MZ and DZ twin correlations for each EF measure and GCA (corrected for age and sex) are displayed in Table 2. The MZ correlations for GCA, WorkMem, and Fluency were significantly larger than the DZ correlations $(p<0.01)$, whereas the differences between $\mathrm{MZ}$ and DZ correlations were non-significant for Flexibility. Table 2 also shows the phenotypic correlations between the four EF variables and GCA. The correlations between EF measures were generally low, ranging from 0.06 to 0.27 , and correlations between Fluency and Inhibition, and Fluency and Flexibility were not significant. Correlations between EF variables and GCA ranged from 0.26 to 0.38 , and were all significant, with the largest correlation being between GCA and WorkMem. The phenotypic correlation between GCA and NART-IQ was moderately high, at 0.60 .

\section{Table 2 about here}

Genetic Modeling

Although the difference between MZ and DZ twin correlations for two of the EF variables, Flexibility and Inhibition, were not significantly different (most likely due to the wide 
confidence intervals), the MZ correlations were significant, and therefore, both variables were included in the model to explore their covariation with the other variables. In examining models containing only A and E components, both the Cholesky and independent pathway models provided a good fit, with the latter providing a slightly better fit (Table 3). Further, we found that all the Environmental (E) cross-paths could be removed simultaneously without significant deterioration of model fit. Fig 1 shows this independent pathway common factor model. For completeness, the Cholesky AE decomposition showing genetic and environmental influences on the relationship between GCA and the four EF measures is shown in Table (a) Appendix 1. Substituting the NART-IQ as an estimate of general intellectual function showed a similar pattern, with all of the covariation between NART-IQ and the EF measures explained by a common genetic factor A1, as displayed in Table (b) Appendix 1.

\section{Table 3 about here}

As can be seen in Fig. 1, the heritability estimate for GCA is high $\left[\left(0.74^{2}\right)+(0.43)^{2}\right)=$ 0.73], moderately high for WorkMem $\left[\left(.52^{2}\right)+\left(.57^{2}\right)=0.59\right]$ and Fluency $(0.63)$, and lower for Inhibition (0.29) and Flexibility (0.31). One common genetic factor accounts for all of the covariance between the four EF variables and GCA. For WorkMem, Fluency, Inhibition and Flexibility, $27 \%, 12 \%, 13 \%$ and $10 \%$ of the variance respectively, was explained by this common genetic factor. This represents 20 to $45 \%$ of the total genetic variance in these measures (i.e. $\left[\left(.52^{2} / 0.59\right)\right]=45 \%$ for WorkMem; $19 \%$ for Fluency; $45 \%$ for Inhibition, $32 \%$ for Flexibility) so that more than half of the genetic variance in WorkMem $\left[\left(.57^{2}\right) / 0.59\right)=$ $54 \%$, Fluency (80\%) and Flexibility (68\%) is due to genetic influences specific to the measure. These common sources of genetic influence are reflected in the genetic correlations 
(Table 4). The genetic correlations were generally larger between the EF and GCA, ranging from 0.36 (Fluency and GCA) to 0.55 (Inhibition and GCA). Genetic correlations between EF measures ranged from 0.52 between WorkMem and Flexibility to 0.36 between WorkMem and Fluency. Genetic correlations between Fluency and both Inhibition and Flexibility were low.

Table 4 about here

Fig. 1 about here

In addition, when we re-ran an AE Cholesky including only the four EF measures (i.e. with GCA removed), a common genetic factor (A1) explained all the significant covariation among the EF measures (Figure 2). Genetic factor loadings for A1 were similar to those found for the independent model (i.e. Confidence Intervals of A1 factor loadings from Fig. 2 overlap with A factor loadings from Fig. 1), and all environmental influences were specific to each measure.

Fig. 2 about here

\section{Discussion}

This study aimed to investigate the genetic influences on four measures of Executive Functions, and their covariation with General Cognitive Ability in older adult twins, aged 65 years and older. In order to achieve this, we used classical twin design and genetic modeling to inform us on the heritability of, the genetic correlations between Working Memory, Verbal 
Fluency, Response Inhibition, and Cognitive Flexibility; and their covariation with General Cognitive Ability. Here we will first compare our findings on the heritability estimates of the EF measures to previous studies, and then the phenotypic and genetic correlations between the EF measures themselves and with GCA. Lastly, we will discuss our findings on the genetic and environmental influences on the neuropsychological measures from genetic modeling.

The heritability estimates of the EF variables differ in that they were higher for Working Memory and Verbal Fluency (approximately 60\%), and lower for Response Inhibition and Cognitive Flexibility (approximately 30\%). The remaining variance represented influences from unique environmental factors (which included measurement error). The difference in heritability of the different EF measures is consistent with the relatively low phenotypic correlations ( 0.06 to 0.27 ) between the various EF measures, and implies that some aspects of executive control are more heritable than others. These data therefore align with the broader literature showing "Executive Function" to be a multi-faceted construct. The heritability estimate of GCA was $73 \%$, which is consistent with the previously reported range of approximately 60 to $80 \%$ of the SATSA studies (Pedersen et al. 1992; McClearn et al. 1997).

Comparing our EF heritability estimates with those of previous aging studies reveals considerable differences, which is likely due to methodological differences. For Digit Span Backwards (Working Memory in our study, both the SATSA (Finkel et al. 1995a; Finkel et al. 1995b, McClearn et al. 1997) and the Longitudinal Study of Aging in Danish Twins (LSADT, McGue and Christensen 2001), the heritability estimates of Digit Span Forward and 
Digit Span Backward (as a measure of memory) ranged from $25 \%$ to $50 \%$ which was lower than our estimate of $60 \%$ for Digit Span Backward alone in our study. As for Fluency, our estimate of $63 \%$ is consistent with Guibilei et al.'s (2008) report of a heritability of $62 \%$, but considerably higher than the estimates of the NHLBI studies. This discrepancy may partly reflect sex differences, as the NHLBI studies included only male participants. In the present study, sex was found to have a significant effect on Fluency, with females performing better than males. Our heritability estimates for Inhibition and Flexibility are considerably lower than those of the NHIBL studies. The latter did not take into account baseline speed in these two measures, and therefore, the disparity in findings here would also be attributable to methodological differences between studies.

It is of note that a review of brain imaging studies of the genetic influences on brain structure has shown very high heritability estimates of $90-95 \%$ for frontal lobe volumes (Peper et al. 2007). Although the heritability of different phenotypes (EF and brain structure) obtained from different samples cannot be compared directly, the difference in the magnitude of these estimates (such as the lower heritability of Response Inhibition and Cognitive Flexibility) raises question of which of these EF measures are indeed functions of the frontal lobes. Alternatively, there may be difference in the genetic influence in different areas of the frontal lobe. The phenotypic correlations amongst the EF measures were generally low, ranging from 0.06 to 0.27 . There is evidence from neuroimaging studies that supports the multidimensional nature of EF. While WorkMem is associated with lateral prefrontal brain structures, switching between tasks (Flexibility) is dependent upon the medial prefrontal cortex, and the ability to inhibit responses (Inhibition) relies on the orbitofrontal cortex (Huizinga et al. 2006). Thus, although each of these executive control processes is subsumed under the frontal system, they are associated with different areas of the frontal lobes. Taken 
together, these results support Salthouse's (2005) contention that EF is not a unitary construct".

As for the phenotypic correlations between the various EF measures and GCA, WorkMem was found to have the largest, albeit modest (0.38) correlation with GCA. This might partly be attributable to their neuroanatomical relationship, with general intellectual functions (verbal reasoning and visual problem resolution in our GCA), and working memory both imposing particular demands upon the lateral prefrontal cortex (Walsh, 1985). However, examination of the confidence intervals of the correlations between GCA and the EF measures show that they all are significant and overlapping. This finding would be inconsistent with Ardila et al.'s (2006) report that there were few significant correlations between IQ scores and EF measures. This would also be inconsistent with the report by Friedman et al. (2006) that "updating", operationalised as working memory in our study, was highly correlated with general intelligence measures, whereas "inhibition" and "shifting" were not.

The extent to which the genetic influence on one measure also impacts another measure is indexed by their genetic correlation. The highest genetic correlation amongst the EF measures was between WorkMem and Flexibility, followed by Inhibition and Flexibility. These moderately high genetic correlations provide some evidence that the EF measures are influenced by common genes, and may have similar biological underpinnings. Taken together, the pattern of results appear to be most parsimoniously explained in the context of the "unity and diversity of Executive Functions" (Miyake et al. 2000), with the genetic 
correlations between the four EF measures and their sharing of one common genetic factor representing "unity". On the other hand, the low phenotypic correlations and the finding that each of the EF measures has their own unique environmental (E) influences would be indicative of their "diversity" (see Miyake et al., 2000 for a further discussion of this issue).

Genetic modeling has shown that the covariance between the five measures (four EF measures and GCA) was entirely due to one common genetic factor shared between them. Similar results emerged with the four-variable AE Cholesky model (with the four EF variables and excluding GCA), and when GCA was replaced by NART-IQ. These results, together with the finding of significant specific environmental influences to each measure but there was no significant shared environmental (E) factor confirmed that the covariation between the EF measures and GCA was entirely explained by genetic influences.

It is of note that while the genetic covariation between EF measures and GCA explained approximately $20-45 \%$ of the total genetic variance of the EF measures, the genetic covariation between the Processing Speed measures and GCA in our previous study using the same sample was considerably larger, explaining $50-77 \%$ of the total genetic variance in four of the five PS measures. The difference in findings of covariation between GCA with the PS and EF domains suggests a much larger genetic overlap between GCA and PS compared to GCA and EF. The low correlation between the different EF in contrast to the PS measures reflects the relative homogeneity of measures within the PS domain and the multi-faceted nature of EF. The contrasting results in examining the genetic influences on these two cognitive domains have potential implications for the study of the two preeminent competing 
theories of cognitive aging: the Processing Speed Theory (Salthouse 1996) and the FrontalExecutive Theory (West 1996).

Our finding indicates that there were environmental influences specific to each of the measures, with the strongest influence (70\%, albeit including measurement error) in Flexibility and Inhibition. It would be worthwhile to investigate the environmental and risk factors involved in these two measures, as they are potentially modifiable. It is also noteworthy that the genetic modeling which used the NART-IQ as a measure independent of executive functions provided results that were consistent with those of the original model (using GCA). This finding provides some support for the NART-IQ, a reading test which has generally been used to estimate pre-morbid intelligence, as a useful alternative measure for current general cognitive functioning in a generally healthy and cognitively intact elderly sample.

The results of this study have several important implications. Firstly, the weak phenotypic correlations between the EF variables and the variability of the heritability of EF measures, supports neuroimaging studies that identify distinct neuroanatomical correlates for different facets of EF, and emphasise the need for different facets of EF to be investigated separately, rather than regarding EF as a global measure subsumed under frontal lobe functions. In addition, the genetic correlations and the covariance found between the EF measures and GCA emphasise the appropriateness of taking EF into account in the study of genetic influence in other cognitive abilities, and vice versa. The considerable difference between the lower heritability estimates of the EF measures in the present study and the very high 
estimates of frontal lobe volume in neuroimaging studies warrants further attention, and the genetic influence on their relationship should be investigated together in a genetically informative sample.

This study complements the limited research in this area of cognitive aging, by being the first to explore the covariation between GCA and EF in older adults and also by using multiple and more valid EF measures that had controlled for speed. Limited by the relatively modest sample size and with the associated lack of statistical power, we could not explore further the genetic relationship between EF and GCA such as sex differences. The inclusion criteria of NART-IQ $\geq 80$ or MMSE $\geq 24$ would also have selected a group of cognitively "healthier" participants, and this may have compromised the generalisability of our findings. Although our results suggest that the EF measures and GCA share the same genetic influence, the direction of causation, that is, the genetic influence on which measure influences the other measure, cannot be determined with our cross-sectional data. We would be able to examine this when longitudinal data become available, and which will also permit us to address the issue of whether the heritability of EF decreases with age.

In summary, our genetic modeling results suggest that the heritability estimates of EF measures of response generation (fluency) and working memory are substantial (approximately 60\%), while those of response inhibition and cognitive flexibility are considerably lower (approximately 30\%). The covariance between the four Executive Function measures and General Cognitive Ability could be entirely explained by shared genetic influences, with no significant environmental influence shared between them. The findings suggest that aspects of EF and measures of GCA share the same genes or set of genes, and merits effort to identify these genes in future studies. 


\section{Acknowledgements}

This research was supported by the National Health and Medical Research Council (ID 401162), and facilitated with access to the Australian Twin Registry, a national research resource supported by an Enabling Grant (ID 310667) from the National Health and Medical Research Council, and administered by The University of Melbourne. We would like to thank the OATS Research Team (www.brainage.med.unsw.edu.au) for their contribution to this study, and most of all, we would like to thank the twins and the informants for their participation in the OATS. 


\section{References}

Arbuthnott K, Frank J (2000) Trail Making Test, Part B as a measure of executive control: validation using a set-switching paradigm. Journal of Clinical and Experimental Neuropsychology 22(4): 518-528

Ardila A, Pineda D, Rosselli M (2000) Correlation between intelligence test scores and executive function measures. Archives of Clinical Neuropsychology 15, 495-513

Baddeley A (1986) Working memory. Oxford: Clarendon Press.

BaddeleyA (1990) Human memory: Theory and practice. London: Erlbaum.

Benton AL, Hamsher KS (1976) Multilingual Aphasia Examination: Manual of instruction University of Iowa, Iowa City

Delis DC, Kaplan E, Kramer JH (2001) Executive Function System: examiner's manual. The Psychological Corporation, San Antonio, USA. 91-103

Bryan J, Luszcz MA (2000) Measurement of executive function: consideration for detecting adult age differences. Journal of Clinical and Experimental Neuropsychology 22(1) 40-55

Finkel D, Pedersen NL, McGue M, McClearn GE (1995a) Heritability of cognitive abilities in adult twins: comparison of Minnesota and Swedish data. Behav Genet 25:421-431

Finkel D, Pedersen NL, McGue M (1995b) Genetic influence on memory performance in adulthood: comparison of Minnesota and Swedish data. Psychol Aging 10: 437-446

Fjell AM, Westlye LT, Amlien E, Espeseth T, Reinvang I, Raz N, Agartz I, Salat DH, Greve DN, Fischl B, Dale AM, Walhovd, KB (2009) High consistency of regional cortical thinning in aging across multiple samples. Cerebral Cortex 19:2001-2012 
Folstein MF, Folstein SE, McHugh PR (1975) "Mini-Mental State” A practical method for grading the cognitive state of patients for the clinician. J Psychiatr Res 12:189-198

Friedman NP, Miyake A, Corley RP, Young SE, DeFries JC, Hewitt JK (2006) Not all executive functions are related to intelligence. Psychological Science, 17(2):172-179

Friedman NP, Miyake A, Young SE, DeFries JC, Corley RP, Hewitt JK (2008) Individual differences in executive functions are almost entirely genetic in origin. Journal of Experimental Psychology: General 137(2):201-225

Giubilei F, Medda E, Fagnani C, Bianchi V, De Carolis, A, Salvetti M, Sepe-Monti M, Stazi MA (2008) Heritability of neurocognitive functioning in the elderly: evidence from an Italian twin study. Age and Aging doi:10.1093/ing/afn132

Head D, Rodrigue KM, Kennedy KM, Raz N (2008) Neuroanantomical and cognitive mediators of -related differences in episodic memory. Neuropsychology 22(4):491-507

Henry JD, von Hippel W, Baynes K (2009) Social inappropriateness, executive control, and aging. Psychology and Aging 24(1):239-244

Huizinga M, Dolan CV, van der Molan MW (2006) -related change in executive function: developmental trends and a latent variable analysis. Neuropsychologia 44(11):2017-2036

Johnson W, Bouchard TJJr, Segal NL, Keyes M, Samuels J. (2003) The Stroop Color-Word Test: genetic and environmental influences: Reading, mental ability, and personality correlates. Journal of Educational Psychology 95:58-65

Kimberg DY, Farah MJ (1993) A unified account of cognitive impairments following frontal lobe dam: the role of working memory in complex, organised behavior. Journal of Experimental Psychology: General 122(4): 411-428 
Lansbergen MM, Kenemans JL, van Engeland H (2007) Stroop interference and attentiondeficit/hyperactivity disorder: a review and meta-analysis. Neuropsychology 21(2):251-262

Lee T, Mosing MA, Henry JD, Trollor JN, Lammel A, Ames A, Martin NG, Wright MJ, Sachdev, PS (2011) Genetic influences on five measures of processing speed and their covariation with general cognitive ability in the elderly: the Older Australian Twin Study. Behav Genet DOI 10.1007/s10519-011-9474-1

Lessov-Schlaggar CN, Swan GE, Reed T, Wolf PA, Carmelli D (2007) Longitudinal genetic analysis of executive function in elderly men. Neurobiol. Aging 28:1759-1768

Lezak MD, Howieson DB, Loring DW (2004) Neuropsychological Assessment (4 $4^{\text {th }}$ ed.) New York:Oxford University Press, Inc.

Luciano M, Gow AJ, Harris SE, Hayward C, Allerhand M, Starr JM, Visscher PM (2009)

Cognitive ability at 11 and 70 years, information processing speed, and APOE variation: the Lothian birth cohort 1936 study. Psychology and Aging 24(1):129-138

McClearn GE, Johansson B, Berg S, Pedersen NL, Ahern F, Petrill SA, Plomin R (1997) Substantial genetic influence on cognitive abilities in twins 80 or more years old. Science 276:1560-1563

McGue M, Christensen K (2001) The heritability of cognitive functioning in very old adults: Evidence from Danish twins d 75 years and older. Psychology and Aging 16(2):272-280

Miyake A, Friedman NP, Emerson MJ, Witzki AH, Howerter A, Wr TD (2000) The unity and diversity of executive functions and their contributions to complex "frontal lobe" tasks: a latent variable analysis. Cognitive Psychology 41:49-100.

Mosocovitch M, Winocur G. (1992) The neuropsychology of memory and aging. In F.I.M. Craik and T.A. Salthouse (Eds), The handbook of aging and cognition (pp. 315-372) 
Neale MC (2005) Twin Studies: Software and Algorithms. Encyclopedia of life sciences. John Wiley and Sons, Ltd. www.els.net

Neale M.C., Boker, SM, Xie, Maes, HH (2002) Mx: statistical modeling. VA 23298: Department of Psychiatry VCU Box 900126, Richmond

Nelson HE and Willison JR (1991). National adult reading test (NART): Test manual ( $2^{\text {nd }}$ ed.). Windsor:NFER-Nelson

Raz N, Gunning-Dixon FM, Head D, Dupuis JH, Acker JD. (1998) Neuroanatomical correlates of cognitive aging: evidence from structural magnetic resonance imaging. Neuropsychology $12: 95-114$

Pedersen NL, Plomin R, Nesselroade J, McClearn GE (1992) A quantitative genetic analysis of cognitive abilities during the second half of the life span. Psychol Science. 3:346-352

Peper JS, Brouwer RM, Boomsma DI, Kahn RS, Hulshoff Pol (2001) Genetic influences on human brain structure: a review of brain imaging studies in twins. Human Brain Mapping. 28:464-473

Plomin R, Pedersen NL, Lichtenstein P, Mcclearn GE (1994) Variability and stability in cognitive abilities are largely genetic later in life. Behav Genet 24:207-215

Purcell S (2008) Statistical methods in behavioral genetics. In Plomin R, Defries JC, McClearn GE, McGuffin P. Behavioral Genetics ( $5^{\text {th }}$ Ed.) New York:Worth Publishers and WH Freeman \& Co

Reitan RM and Wolfson D (1985) The Halstead-Reitan neuropsychological test battery: theory and interpretation. Tucson AZ:Neuropsychology Press

Rey A (1964) L'examen clinique en psychologie. Presses Universitaires de France, Paris 
Sachdev PS, Lammel A, Trollor JN, Lee T, Wright MJ, Ames D, Wen W, Martin NG, Brodaty H, Schofield PR, and the OATS research team (2009) A Comprehensive Neuropsychiatric Study of Elderly Twins: The Older Australian Twins Study. Twin Research and Human Genetics 12(6): 573-582

Sachdev PS, Brodaty, H., Reppermund, S., Kochan, N. A., Trollor, J. N., Draper, B., ...\& Memory and Aging Study Team. (2010) Methodology and baseline medical and neuropsychiatric characteristics of an elderly epidemiological non-demented cohort of Australians aged 70-90 years. International Psychogeriatrics, 22(8), 1248-64.

Salthouse TA (2005) Relations between cognitive abilities and measures of executive functioning. Neuropsychology 19(4):532-545

Salthouse, T. A. (1996). The processing-speed theory of adult age differences in cognition. Psychological Bulletin, 103, 403-428.

Shallice T (1988) From neuropsychology to mental structure. Cambridge: Cambridge University Press

Sivan AB, Spreen O (1996) Der Benton-Test ( $7^{\text {th }}$ ed.). Berne, Switzerland: Verlag Hans Huber Smith EE, Jonides J (1999) Stor and executive processes in the frontal lobes. Science 283: 16571661

Snow WG, Weinstock J (199) Sex differences among non-brain-damaged adults on the Spinath Weschsler audlt intelligence scales: a review of the literature. J Clin Exp Neuropsychol 12 (6):873-886

Stins JF, van Baal GCM, Polderman TJC, Verhulst FC, Boomsma DI (2004) Heritability of Stroop and flanker performance in 12-year old children. BMC Neuroscience 5:49 
Swan GE, Carmelli D (2002) Evidence of genetic mediation of executive control: a study of aging male twins. The Journal of Gerontology 57B(2):133-143

Taylor J (2007) Heritability of Wisconsin Card Sorting Test (WCST) and Stroop Color-word Test performance in normal individuals: implications for the search for endophenotypes. Twin Research and Human Genetics 10(6):829-834

Wechsler D (1987) Wechsler Memory Scale-Revised manual. The Psychological Corporation. San Antonio

Wechsler D (1997) Adult intelligence scale, 3rd edn. The Psychological Corporation, San Antonio

West RL (1996) An application of prefrontal cortex function theory to cognitive aging. Psychological Bulletin 120:272-292 


\section{Figure Legend}

Fig.1 Best fitting independent pathway model showing genetic and environmental influences on the relationship between General Cognitive Ability (GCA), working memory (WorkMem), verbal fluency (Fluency), Inhibition, and Flexibility. Non-significant specific pathways in the model $(p>0.05)$ were retained for completeness and are shown as dashed lines. $\mathrm{A}=$ common genetic factor, $\mathrm{As}=$ genetic influence specific to variable, $\mathrm{Es}=$ unique environmental influence specific to variable. 


\section{Figure Legend}

Fig. 2. Cholesky decomposition showing genetic and environmental influences on the relationship between WorkMem, Fluency, Inhibition, and Flexibility. Non-significant pathways in the model $(p>0.05)$ were retained for completeness and are shown as dashed lines. 


\section{APPENDIX 1}

Table (a) Cholesky AE decomposition showing genetic (A) and environmental (E) influences on the relationship between GCA, WorkMem, Fluency, Inhibition, and Flexibility

\begin{tabular}{lcclcc}
\hline A Paths & GCA & WorkMem & Fluency & Inhibition & Flexibility \\
\hline A Paths & NART-IQ & WorkMem & Fluency & Inhibition & Flexibility \\
\hline A1 & $.86(.80, .90)^{*}$ & $.40(.28, .50)^{*}$ & $.29(.16, .42)^{*}$ & $.31(.16, .44)^{*}$ & $.27(.14, .39)^{*}$ \\
\hline A1 & $.90(.86, .92)^{*}$ & $.44(.34, .53)^{*}$ & $.32(.20, .43)^{*}$ & $.39(.26, .50)^{*}$ & $.20(.08, .31)^{*}$ \\
A2 & & $.66(.56, .74)^{*}$ & $.17(.01, .32)^{*}$ & $.07(-.11, .25)$ & $.18(.03, .34)^{*}$ \\
A2 & & $-.64(-.71,-.54)$ & $-.15(-.29, .0)$ & $-.03(-.21, .14)$ & $-.23(-.39,-.07)$ \\
A3 & & $.73(.64, .80)^{*}$ & $-.06(-.23, .12)$ & $-.13(-.29, .03)$ \\
A3 & & $.73(.65, .79)^{*}$ & $-.06(-.22, .11)$ & $-.10(-.25, .05)$ \\
A4 & & & $.45(.17, .45)^{*}$ & $.08(-.25, .42)$ \\
& & & & $.43(-.59, .59)$ \\
A5 & & & & \\
\hline
\end{tabular}

\section{E Paths}

E1

$.01(-.10, .13)$

$.09(-.06, .25)$

$.04(-.10, .19)$

E2

$.63(.56, .72)^{*}$

$.08(-.03,18)$

$-.08(-.23, .08)$

$-.12(-.26, .01)$

E3

$.59(.51, .68)^{*}$

$.01(-.16, .17)$

$.09(-.05,-23)$

E4

$.82(.72, .93) *$

$0(-.16, .14)$

E5

$.81(.73, .90)^{*}$ 
$.44(-.60, .60)$

\begin{tabular}{|c|c|c|c|c|c|}
\hline EPaths & Twilferre & elations & (Wfate & Phenotypie effrelat & ТӨРАҢ \\
\hline & $\mathrm{MZ}(186-254)_{54} \mathrm{D}$ & $\mathrm{DZ}(154-218)$ & WorkMem & Fluency 226 & Flexibility \\
\hline E1 & $.44(.38, .51)^{*}$ & $.05(-.05, .17)$ & $.11(0, .22)$ & $.04(-.13, .21)$ & $.08(-.06, .22)$ \\
\hline $\begin{array}{l}\text { GCA (IQ) } \\
\text { E2 }\end{array}$ & $98.86(14.77)$ & $\begin{array}{c}101.37(15.19) \\
.63(.56, .71)^{*}\end{array}$ & $\begin{array}{c}100.84(14.84) \\
.06(-.04, .16)\end{array}$ & $\begin{array}{l}99.61(15.08) \\
-.09(-.24, .07)\end{array}$ & $\begin{array}{l}100.11(14.98) \\
-.13(-.26,0)\end{array}$ \\
\hline $\begin{array}{l}\text { WorkMem } \\
\text { E3 }\end{array}$ & $4.68(1.27)$ & $4.76(1.26)$ & $\begin{array}{c}4.90(1.38) \\
.57(.50, .66)^{*}\end{array}$ & $\begin{array}{l}4.63(1.19) \\
-.02(-.18, .15)\end{array}$ & $\begin{array}{r}4.72(1.26) \\
.06(-.08, .20)\end{array}$ \\
\hline Fluency & $38.17(12.21)$ & $36.67(12.47)$ & $36.72(12.64)$ & $37.83(12.21)$ & $37.48(12.34)$ \\
\hline E4 & & & & $.82(.72, .92)^{*}$ & $-.02(-.16,-.13)$ \\
\hline Inhibition & $1.95(0.43)$ & $1.94(0.41)$ & $1.96(0.51)$ & $1.94(0.45)$ & $1.94(0.47)$ \\
\hline E5 & & & & & $.80(.72, .89) *$ \\
\hline Flexibility & $2.74(0.95)$ & $2.57(0.82)$ & $2.65(0.99)$ & $2.67(0.84)$ & $2.66(0.89)$ \\
\hline
\end{tabular}

APPENDIX 1

Table (b) Best fitting Cholesky decomposition showing genetic (A) and environmental (E) influences on the relationship between NART-IQ, WorkMem, Fluency, Inhibition, and Flexibility

$*$ denotes significant paths $(\mathrm{p}<.05)$

Table 1 Means (and standard deviations) of General Cognitive Ability (GCA) and four Executive Function measures for Monozygotic (MZ) and Dizygotic (DZ) twins, males and females, and total sample

GCA: General Cognitive Ability, WorkMem:Digit Span Backward, Fluency: Controlled Oral Word Association Test, Inhibition: Stroop3/1 ratio score, Flexibility:TMTB/A ratio score

Table II. Maximum Likelihood Estimation of Twin Correlations and Phenotypic Correlations between the four Executive Function variables and General Cognitive Ability (GCA), corrected for age and sex with $95 \%$ confidence intervals in parentheses. 


\begin{tabular}{|c|c|c|c|c|c|c|}
\hline GCA & $.74(.64 ; .81)$ & $.29(.08 ; .46)$ & $.38(.29 ; .46)$ & $.26(.16 ; .30)$ & $31(.20 ; .41)$ & $.26(.16 ; .35)$ \\
\hline WorkMem & $.59(.46 ; .69)$ & $.28(.10 ; .45)$ & & $.27(.18 ; .36)$ & $.13(.12 ; .24)$ & $.15(.06 ; .24)$ \\
\hline Fluency & $.66(.55 ; .75)$ & $.13(-.07 ; .32)$ & & & $.06(-.06 ; .18)$ & $.06(-.04 ; .16)$ \\
\hline Inhibition & $.29(.08 ; .47)$ & $.10(-.16 ; .34)$ & & & & $.15(.04 ; .25)$ \\
\hline Flexibility & $.31(.13 ; .47)$ & $.22(.01 ; .47)$ & & & & \\
\hline
\end{tabular}

Table III. Model fitting results for the five variables with the best fitting model in bold. Model fit ($2 \mathrm{LL}$ ) of the independent pathway model was compared to the Cholesky (AE) decomposition.

\begin{tabular}{|c|c|c|c|c|c|c|}
\hline Multivariate model fitting results & AIC & $-2 L L$ & df & $\begin{array}{c}\Delta- \\
2 \mathrm{LL}\end{array}$ & $\begin{array}{l}\Delta- \\
\mathrm{df}\end{array}$ & $\begin{array}{l}p \\
\text { value }\end{array}$ \\
\hline Model 1. Cholesky ACE decomposition & 1445.98 & 5688.50 & 2118 & & & \\
\hline Model 2. Cholesky AE decomposition & 1424.79 & 5690.79 & 2133 & 2.29 & 15 & 0.99 \\
\hline Model 3. Omnibus drop of all E cross paths of model 2 & 1416.74 & 5702.74 & 2143 & 11.95 & 10 & 0.29 \\
\hline Model 4. Independent pathway model -1 common genetic factor ( $A E$ ) & 1422.22 & 5698.12 & 2138 & 7.33 & 5 & 0.20 \\
\hline Model 5. Omnibus drop of all E cross paths of model 4 & 1417.24 & 5713.24 & 2148 & 15.12 & 10 & 0.13 \\
\hline
\end{tabular}


Table IV. Genetic correlations between Executive Functions variables and General Cognitive Ability

\begin{tabular}{lcccc}
\hline & GCA & WorkMem & Fluency & Inhibition \\
\hline WorkMem & .51 & & & \\
Fluency & .36 & .36 & & \\
Inhibition & .55 & .39 & .14 & \\
Flexibility & .48 & .52 & .04 & .48 \\
\hline
\end{tabular}

